\title{
Pollen-derived nonallergenic substances enhance Th2-induced IgE production in B cells
}

\author{
S. Oeder ${ }^{1,2}$, F. Alessandrini ${ }^{1,2}$, O. F. Wirz ${ }^{3}$, A. Braun ${ }^{1,4}$, M. Wimmer ${ }^{1,2,5}$, U. Frank ${ }^{2,6}$, M. Hauser ${ }^{2,7}$ \\ J. Durner ${ }^{6}$, F. Ferreira ${ }^{7}$, D. Ernst ${ }^{6}$, M. Mempel ${ }^{1,4}$, S. Gilles ${ }^{1,2,5}$, J. T. M. Buters ${ }^{1,2}$, H. Behrendt ${ }^{1,2}$, \\ C. Traidl-Hoffmann ${ }^{1,2,5}$, C. Schmidt-Weber', M. Akdis ${ }^{3, *}$ \& J.'Gutermuth ${ }^{1,8, *}$ \\ ${ }^{1}$ Center of Allergy and Environment (ZAUM), Technische Universität München and Helmholtz Zentrum München, Member of the German \\ Center for Lung Research (DZL), Munich, Germany; ${ }^{2}$ Christine Kühne - Center for Allergy Research and Education, CK-CARE; ${ }^{3}$ Swiss \\ Institute of Allergy and Asthma Research (SIAF), Davos, Switzerland; ${ }^{4}$ Department of Dermatology, Venereology and Allergology, University \\ Medical Center, Georg August University, Göttingen; ${ }^{5}$ nstitute of Environmental Medicine, UNIKA-T, Technische Universität München, \\ Munich; ${ }^{6}$ Institute of Biochemical Plant Pathology, Helmholtz Center Munich, Neuherberg, Germany; ${ }^{7}$ Department of Molecular Biology, \\ University of Salzburg, Salzburg, Austria; ${ }^{8}$ Department of Dermatology, Vrije Universiteit Brussel, Brussels, Belgium
}

\section{Keywords}

adjuvant; B cell; lgE; phytoprostane E1; pollen.

\section{Correspondence}

Dr. Sebastian Oeder, Center for Allergy and Environment (ZAUM), Technische Universität München and Helmholtz Zentrum München, Member of the German Center for Lung Research (DZL), Biedersteiner Str. 29, 80802 Munich, Germany. Tel.: +498941403477

Fax: +498941403452

E-mail: sebastian.oeder@Irz.tum.de

*These authors contributed equally.

Each named author contributed to the manuscript.

DOI:10.1111/all.12707

\begin{abstract}
Background: B cells play a central role in IgE-mediated allergies. In damaged airway epithelium, they are exposed directly to aeroallergens. We aimed to assess whether direct exposure of B cells to pollen constituents affects allergic sensitization.

Methods: B cells from murine splenocytes and from blood samples of healthy donors were incubated for 8 days under Th2-like conditions with aqueous ragweed pollen extracts (Amb-APE) or its constituents. Secreted total IgM, IgG, and IgE was quantified by ELISA. Additionally, birch, grass, or pine-pollen extracts were tested. The number of viable cells was evaluated by ATP measurements. Bcell proliferation was measured by CFSE staining. IgE class switch was analyzed by quantitation of class switch transcripts. In an OVA/Alum i.p.-sensitization mouse model, Amb-APE was intranasally instilled for 11 consecutive days.

Results: Upon Th2 priming of murine B cells, ragweed pollen extract caused a dose-dependent increase in IgE production, while IgG and IgM were not affected. The low-molecular-weight fraction and phytoprostane E1 (PPE1) increased IgE production, while Amb a 1 did not. PPE1 enhanced IgE also in human memory B cells. Under Th1 conditions, Amb-APE did not influence immunoglobulin secretion. The IgE elevation was not ragweed specific. It correlated with proliferation of viable B cells, but not with IgE class switch. In vivo, Amb-APE increased total IgE and showed adjuvant activity in allergic airway inflammation.

Conclusions: Aqueous pollen extracts, the protein-free fraction of Amb-APE, and the pollen-contained substance PPE1 specifically enhance IgE production in Th2primed B cells. Thus, pollen-derived nonallergenic substances might be responsible for B-cell-dependent aggravation of IgE-mediated allergies.
\end{abstract}

\section{Abbreviations}

Amb, Ambrosia; APE, aqueous pollen extract; BAL, bronchoalveolar lavage; Bet, Betula; CSR, class switch recombination; OVA, ovalbumin; PGE2, prostaglandin E2; Phl, phleum; Pin, pinus; PPE1, phytoprostane E1; Th2, T helper cell 2.
Immunoglobulin E (IgE) is a fundamental player in atopic diseases and a hallmark of allergic sensitization (1). Upon type $2 \mathrm{~T}$-cell help, B cells proliferate, undergo immunoglobulin isotype class switch recombination (CSR) toward IgE, and differentiate into antibody-secreting plasma cells. IgE is necessary for immediate phase reactions like mast cell and basophil degranulation. In damaged airway epithelium, B 
cells can be exposed directly to aeroallergens, and despite their central role in allergic diseases, little is known about how B lymphocytes react upon exposure to pollen constituents.

In the past, we have shown that pollen grains not only are carriers of allergen, but also contain bioactive pollen-associated lipid mediators (PALMs) and other immunomodulatory substances (2). Therefore, allergic sensitization is not only due to genetic predisposition and molecular features of allergens, but is also facilitated by exposure to environmental cofactors that break immunotolerance (3-5): (i) Proinflammatory PALMs (e.g. oxylipins) exert chemoattractive effects on human neutrophils (6) and eosinophils (7) in vitro. (ii) Immunomodulatory PALMs such as E1 phytoprostanes generate a Th2-favouring milieu that is inhibitory for the synthesis of Th1-type chemokines by human dendritic cells (8-10). (iii) NADPH oxidases generate reactive oxygen species that damage airway epithelium and increase allergic lung inflammation $(11,12)$.

IgE is not only produced centrally, but also locally, and B cells undergo IgE CSR in nasal and mucosal tissue (13, 14). The concept of 'local allergic rhinitis' (LAR) with local IgE production in the airway mucosa suggests to analyze the direct influence of pollen extracts on B lymphocytes $(15,16)$. CSR requires the deletion of genomic DNA, containing constant gene regions of the $\operatorname{IgM} / \mathrm{IgG}$ heavy chain, which is mediated by activation-induced cytidine deaminase (AID) and the sterile $\varepsilon$-germline transcript (17-19). IgE CSR results in an $\varepsilon$-postswitch transcript, a precursor of IgE (20-22). An unresolved question is whether adjuvants can facilitate CSR and thereby aggravate allergic diseases. Therefore, we analyzed the impact of pollen-derived extracts on T-cell-initiated CSR and subsequent IgE production.

The pollen extracts we used in our study originated from the highly allergenic plant species ragweed (Ambrosia artemisiifolia), birch (Betula verrucosa), and timothy grass (Phleum pratense), and from less allergenic pine (Pinus strobus). Additionally, protein-free fraction $(<3 \mathrm{kDa})$ of ragweed pollen extract, which contains PALMs and other low-molecular-weight molecules, was generated (23-25).

In this study, we demonstrate that pollen extracts from various plant species enhance Th2-induced production of total IgE. Due to their capacity to specifically increase IgEproducing $B$ cells, pollen-derived $\mathrm{E} 1$ phytoprostane might be a candidate substance responsible for the aggravation of $\operatorname{IgE}$ production. The IgE-adjuvant activity of ragweed pollen extract was verified in vivo by exacerbation of a mouse model of OVA-induced allergic lung inflammation.

\section{Materials and methods}

\section{Preparation of pollen extracts}

Aqueous pollen extracts (APE) of ragweed, birch, grass, and pine were generated from pollen grains (Allergon, Ängelholm, Sweden) incubated in PBS $(10 \mathrm{mg} / \mathrm{ml})$ for $30 \mathrm{~min}$ at $37^{\circ} \mathrm{C}$. After pelleting ( $20 \mathrm{~min}$ at $3.345 \mathrm{~g}$ ), supernatants were sterile-filtered ( $0.2 \mu \mathrm{m}$; Millipore, Schwalbach, Germany). To obtain a protein-free fraction, the extracts were passed three times over a 3-kDa cutoff filter (Amicon Ultra YM3; Millipore). The absence of proteins in Amb-APE $<3 \mathrm{kDa}$ was verified by Coomassie Assay (Invitrogen, Karlsruhe, Germany). LPS was measured by LAL assay and was below $1 \mathrm{EU} / \mathrm{ml}$. Natural Amb a 1 was purified by ion exchange chromatography. See Data S1 for more details.

\section{Animals}

Female, 6- to 10-week-old C57BL/6 and BALB/c mice were obtained from Charles River (Sulzfeld, Germany), housed under specific pathogen-free conditions in individually ventilated cages (VentiRack; BioZoneGlobal Ltd., Ramsgate, UK), and fed by standard pellet diet and water ad libitum. The study was conducted under federal guidelines for the use and care of laboratory animals and was approved by the Government of the District of Upper Bavaria and the Animal Care and Use Committee of the Helmholtz Center Munich (Munich, Germany).

\section{Sensitization protocol}

An established OVA-sensitization model was used $(26,27)$. Briefly, 6-week-old BALB/c mice were sensitized by intraperitoneal injections of $1 \mu \mathrm{g}$ OVA (grade VI; Sigma-Aldrich, Munich, Germany) in $200 \mu \mathrm{l}$ PBS adsorbed to $2.5 \mathrm{mg}$ aluminum hydroxide (alum) (Thermo Fischer Scientific, Waltham, MA, USA) on days $0,7,14$, and 42 . Nonsensitized (NS) mice received PBS only in alum. From day 43 onward, mice were instilled on eleven consecutive days with $20 \mu 1$ APE of ragweed $(10 \mathrm{mg} / \mathrm{ml})$ or PBS, followed by one aerosol challenge on day 52 for 20 min with $1 \%$ ovalbumin delivered by a Pari-Boy nebulizer (Pari, Starnberg, Germany). Bronchoalveolar lavage (BAL) analysis was performed $24 \mathrm{~h}$ (day 53) or 7 day (day 59) after OVA challenge. Blood samples were taken before sensitization and at the end of the experiment.

\section{Analysis of bronchoalveolar lavage, lung histology, and serology}

Bronchoalveolar lavage, lung histology, and immunoglobulin determination in plasma were performed as described previously $(26,27)$. See Data S1 for more details.

\section{Murine B-cell isolation, culture, and stimulation}

Spleens were taken aseptically from female, 7- to 10-week-old C57BL/6 mice, and single-cell suspensions were generated. Resting (mainly naïve) B cells were isolated by untouched magnetic cell sorting (MACS, B-cell Isolation Kit; Miltenyi, Bergisch Gladbach, Germany). Purity (anti-B220 staining) reached at least $97 \%$. B cells were cultured in complete RPMI containing $2 \mathrm{mM}$ glutamine, $100 \mathrm{U} / \mathrm{ml}$ penicillin, $100 \mu \mathrm{g} / \mathrm{ml}$ streptomycin, $50 \mu \mathrm{M} \beta$-mercaptoethanol, $1 \mathrm{mM}$ minimum essential media (MEM) sodium pyruvate, $0.1 \mathrm{mM}$ 
MEM nonessential amino acids, $20 \mathrm{mM}$ HEPES, and $10 \%$ FCS (PAA, Coelbe, Germany) in triplicates during polyclonal stimulation with $5 \mu \mathrm{g} / \mathrm{ml}$ anti-CD40 $\mathrm{mAb}$ (eBioscience, San Diego, CA, USA) and $25 \mathrm{ng} / \mathrm{ml}$ murine IL-4 (PeproTech, Rocky Hill, NJ, USA) for a Th2-like condition or $25 \mathrm{ng} / \mathrm{ml}$ IFN- $\gamma$ (PeproTech) for a Th1-like condition. Additionally, B cells were exposed to pollen extracts, Amb a 1, PPE1, or PGE2 for 8 days.

\section{Human B-cell isolation, culture, and stimulation}

Peripheral blood mononuclear cells were isolated from peripheral venous blood of healthy donors (commercially available buffy coats) by Ficoll (Biochrom, Berlin, Germany) density gradient centrifugation. Total $\mathrm{CD} 19^{+} \mathrm{B}$ cells were isolated using untouched MACS sorting (B-cell Isolation Kit II human; Miltenyi). Purified B cells were stained with anti-CD19-APCCy 7 and anti-CD27-PE (Biolegend, San Diego, CA, USA), and naïve $\left(\mathrm{CD} 19^{+} \mathrm{CD} 27^{-}\right)$and memory $\left(\mathrm{CD} 19^{+} \mathrm{CD} 27^{+}\right) \mathrm{B}-$ cell subsets were sorted using FACSAria II cell sorter (BD Biosciences, Franklin Lakes, NJ, USA). B cells were cultured in RPMI1640 medium supplemented with $1 \mathrm{mM}$ sodium pyruvate, $1 \%$ MEM nonessential amino acids and vitamins, $2 \mathrm{mM}$ L-glutamine, $100 \mathrm{U} / \mathrm{ml}$ penicillin, $100 \mathrm{mg} / \mathrm{ml}$ streptomycin, $50 \mathrm{mM}$ 2-ME (GIBCO BRL, Basel, Switzerland), and 10\% heat-inactivated FCS (Sera-Lab Ltd., Sussex, UK).

A total of 1000 naïve B cells and 5000 memory B cells were stimulated with human CD40-ligand-presenting mouse fibroblasts, $25 \mathrm{ng} / \mathrm{ml}$ human IL-21 (Novartis, Basel, Switzerland) and human IL-4 (Novartis), and increasing concentrations of Amb-APE or PPE1 for 12 days. To optimize the induction of $\operatorname{IgE}$ in our experiments, a titration of naïve and memory B cells was done prior to the stimulation experiment starting with 25000 cells down to 195 cells per well in a 96 well plate (Fig. S2) (28).

\section{Immunoglobulin determination}

Total IgE, IgG, and IgM in cell culture supernatants were measured by ELISA (Jackson ImmunoResearch, West Grove, IA, USA), according to the manufacturer's instructions. See Data $\mathbf{S} 1$ for more details.

\section{Murine B-cell proliferation and apoptosis assays}

B-cell count was assessed by measuring intracellular ATP (CellTiter-Glo ${ }^{\circledR}$; Promega Inc., Mannheim, Germany). Intracellular ATP concentration was correlated with viable B-cell count (trypan blue-negative cells in Neubauer chamber; data not shown). Proliferation was tested by CFSE dilution (flow cytometry) and apoptosis by annexin $\mathrm{V}$ and 7-AAD staining. Percentage of apoptotic single annexin V-positive cells was determined by flow cytometry.

\section{RNA extraction and real-time PCR}

Total RNA extraction of murine B cells and real-time PCR were performed according to the manufacturer's instructions
(RNeasy Mini Kit; Qiagen, Hilden, Germany). Expression of IgE class switch-associated transcripts AID (activationinduced cytidine deaminase), $\varepsilon$-GLT (germline transcript), and $\varepsilon$-PST (postswitch transcript) was assessed. Relative mRNA expression levels were calculated using the $2^{-\Delta \Delta C t}$ method. Hypoxanthine guanine phosphoribosyl transferase (HPRT) was used as housekeeping gene. Primer sequences are listed in Table S1.

\section{Data analysis}

Results are shown as boxplots indicating minimum, $25 \%$ percentile, median, $75 \%$ percentile, and maximum or as bar graphs indicating mean \pm standard deviation. Statistical significance was determined by paired student's $t$-test if not stated otherwise. Results were considered significant as $P \leq 0.05 ;{ }^{*} P \leq 0.05 ;{ }^{* *} P \leq 0.01 ; * * * P \leq 0.001$.

\section{Results}

Aqueous ragweed pollen extract (Amb-APE) enhances IgE production in Th2-stimulated murine B cells

To mimic Th2-mediated activation and subsequent IgE production, MACS-sorted naïve B cells from murine C57BL/6 splenocyte suspensions were stimulated for 8 days with antiCD40 and IL-4 or medium control. The influence of ragweed pollen on $\mathrm{T}$ helper 2 cell-induced IgE production was analyzed by an additional exposure to Amb-APE (Fig. 1A).

Without Th2 stimulation, Amb-APE had no effect on immunoglobulin production. Neither IgE, nor IgG or IgM levels were increased in supernatants from B-cell cultures (Fig. 1B). However, when anti-CD40/IL-4 was added, AmbAPE dose-dependently enhanced the production of IgE (Fig. 1C). In contrast, Amb-APE did not increase the production of IgG or IgM. Thus, under Th2 conditions AmbAPE selectively enhanced IgE production. When B cells were stimulated with the IgG-promoting Thl cytokine IFN- $\gamma$ (instead of IL-4), IgG, IgM, and IgE production were not enhanced (Fig. 1D).

\section{Intranasal instillation of Amb-APE elevates levels of total IgE in plasma of OVA-sensitized mice}

The IgE-enhancing effects seen in vitro prompted us to analyze whether these effects have physiological relevance in vivo. Thus, OVA-sensitized BALB/c mice were instilled intranasally with Amb-APE or PBS for eleven consecutive days. Nonsensitized mice instilled with Amb-APE were used as additional controls. On the last day of intranasal instillation, all mice were challenged with OVA aerosol (Fig. 2A). One or seven days after the aerosol challenge, plasma was analyzed for total IgE levels. In nonsensitized mice, instillation of Amb-APE followed by OVA-aerosol challenge had no influence on total IgE levels (NS/APE/OVA; Fig. 2B). In OVA-sensitized mice, intranasal instillation of Amb-APE followed by OVA-aerosol challenge (S/APE/OVA) resulted in significantly increased total IgE levels compared to 

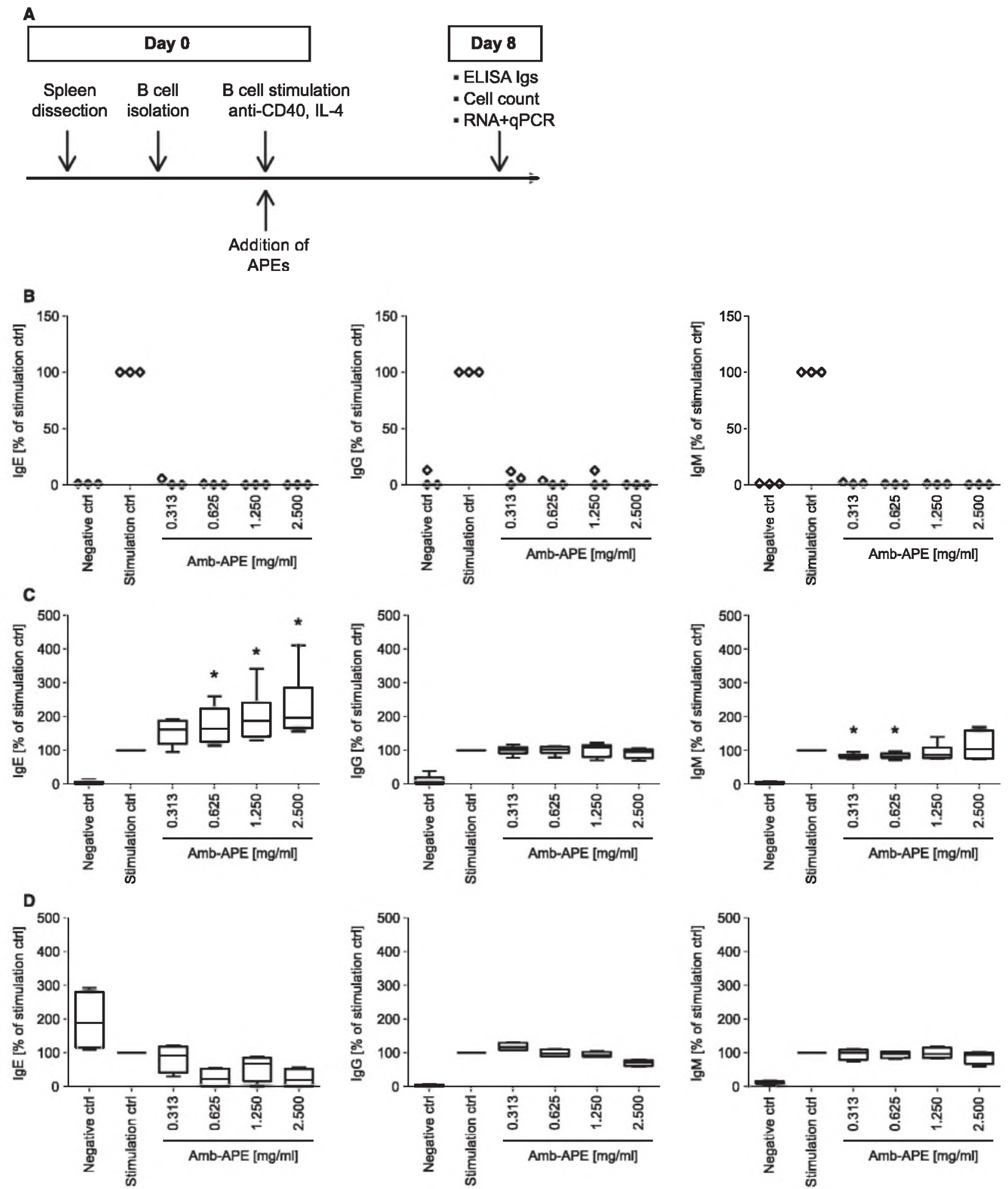

Figure 1 Experimental setup and lgE-enhancing effect of Amb-APE. (A) Total $\operatorname{lgE}$, $\lg G$, and $\lg M$ were measured on day 8 after exposure of naive $B$ cells from C57BL/6 mice to increasing concentrations of Amb-APE without (B) or with stimulation with anti-CD40 and IL-4 (C) or with stimulation with anti-CD40 and IFN- $y$ (D). Negative ctrl. = untreated cells, stimulation ctrl. $=$ cells stimulated with anti-CD40 and
IL-4 (B, C) or anti-CD40 and IFN- (D). Absolute values: (C) $579 \mathrm{ng} / \mathrm{ml}$ lgE, $37.4 \mathrm{ng} / \mathrm{ml} \mathrm{lgG,} 2520.6 \mathrm{ng} / \mathrm{ml} \mathrm{lgM}$; (D) $1 \mathrm{ng} / \mathrm{ml} \mathrm{lgE,} 52.7 \mathrm{ng} / \mathrm{ml}$ $\mathrm{lgG}, 1213.3 \mathrm{ng} / \mathrm{ml} \mathrm{lgM}$. Data are shown as individual data points $(B$, D) or medians \pm quartiles and maximum/minimum. $n=3$ (B), 6 (C), or 4 (D) independent experiments. ${ }^{*} P \leq 0.05$ compared to stimulation control (Wilcoxon's signed-rank test). 

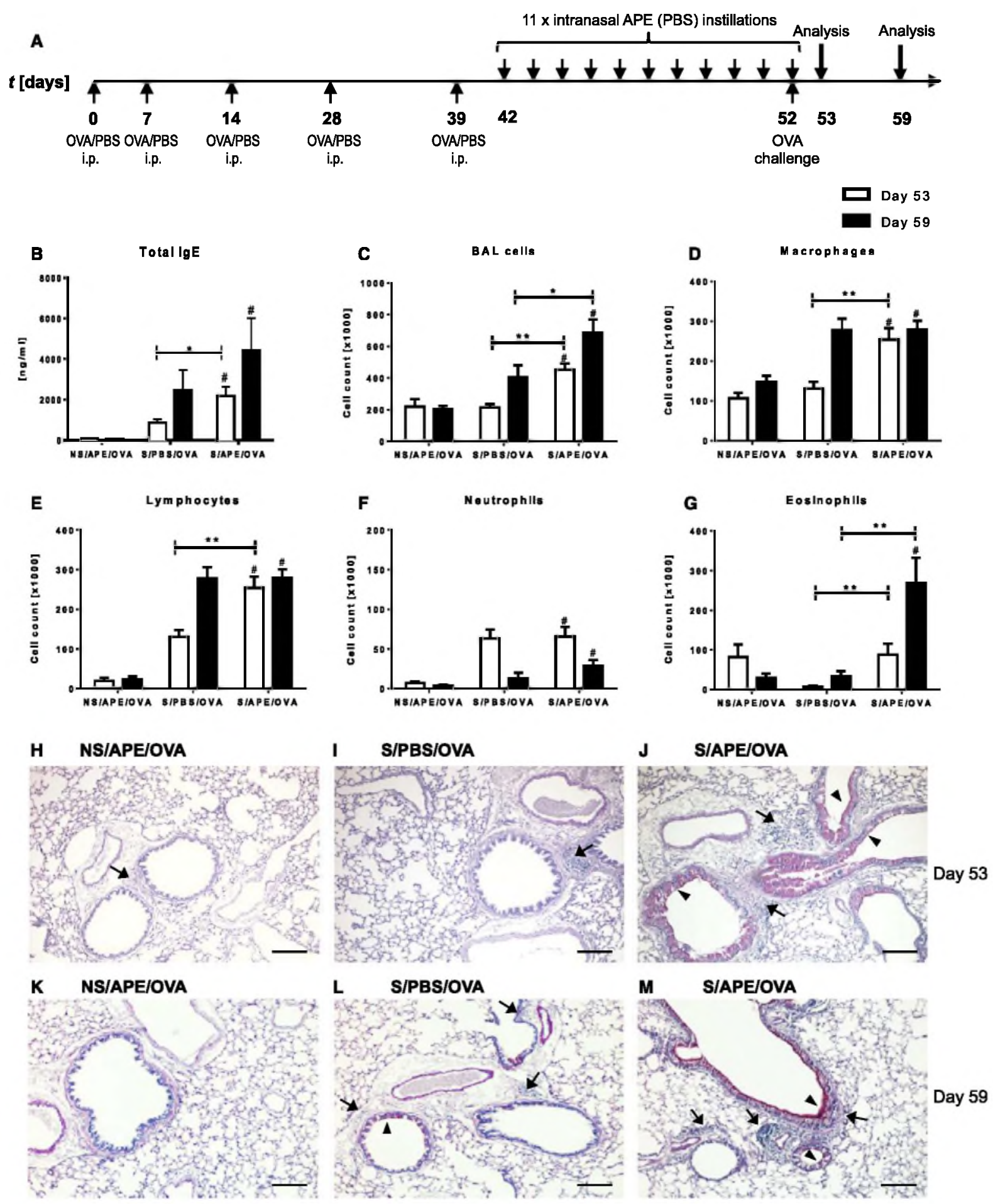

Figure 2 Adjuvant activity of intranasal Amb-APE on OVA-specific ainway inflammation. (A) BALB/c mice were i.p. sensitized with OVA/alum (S) or control solution (PBS/alum [NS]). Prior to OVAaerosol challenge on day 52, mice received i.n. Amb-APE (APE) or PBS for eleven consecutive days. Mice were killed 1 day (day 53) or 7 days (day 59) after OVA challenge. Levels of total lgE in plasma (B), BAL total cell count (C), total macrophages (D), lympho- cytes (E), neutrophils (F), and eosinophils (G) at day 53 (white bars) and at day 59 (black bars). Data are expressed as mean + SEM. $n=6-12$ mice per group. ${ }^{\#} P<0.05$ vs NS/APE/OVA; ${ }^{*} P \leq 0.05$, ${ }^{*} P \leq 0.01$. Representative pulmonary sections (PAS-staining) at day $53(\mathrm{H}-\mathrm{J})$ and at day $59(\mathrm{~K}-\mathrm{M})$. Arrows: inflammatory infiltrate; arrowheads: mucus hypersecretion; scale bar: $100 \mu \mathrm{m}$. 
nonsensitized mice ( $P<0.05$ vs NS/APE/OVA, Fig. 2B) and compared to PBS instillation in the early time-point $(P<0.05$ vs S/PBS/OVA for day 53). In the late time-point (day 59), the increase of total IgE in S/APE/OVA vs S/PBS/ OVA did not reach statistical significance (Fig. 2B).

\section{Intranasal instillation of Amb-APE aggravates OVA-specific lung inflammation in OVA-sensitized mice}

In OVA-sensitized BALB/c mice, intranasal instillation of Amb-APE followed by OVA challenge caused a significant increase in inflammatory lung cell infiltrate as analyzed by bronchoalveolar lavage compared to PBS treatment $(P<0.01$ and $P<0.05$ vs S/PBS/OVA for days 53 and 59, respectively; Fig. 2C). The aggravated response in S/APE/ OVA was characterized by significantly elevated numbers of macrophages (Fig. 2D), lymphocytes (Fig. 2E), and eosinophils (Fig. 2G) on day 53, which increased even more on day 59 compared to S/PBS/OVA. In contrast, neutrophil numbers in S/APE/OVA increased slightly, but not significantly, only on day 59 compared to S/PBS/OVA (Fig. 2F).

The exacerbation of lung inflammation was also evident in the histopathological analysis of lung specimen. NS/APE/ OVA at day 53 showed a slight inflammatory cell infiltration, which disappeared completely by day 59 (Fig. $2 \mathrm{H}, \mathrm{K}$, respectively). S/PBS/OVA showed a mild inflammatory infiltrate at both time-points and mucus hypersecretion at day 59, although both to a lesser extent compared to S/APE/ OVA (Fig. 2I,L). In sensitized mice, intranasal instillation of total Amb-APE followed by a single OVA challenge leads to exacerbated and persisting peribronchial and perivascular inflammation and strong mucus hypersecretion (S/APE/OVA, Fig. 2J,M). This finding indicates an adjuvant activity of Amb-APE on an unrelated bystander inflammation.
Protein-free pollen fractions are sufficient to increase Th2-induced IgE in vitro

Pollen extracts increased total IgE in a dose-dependent manner in isolated B cells from C57BL/6 mice (Fig. 1). Nevertheless, we could not detect Amb a 1-specific IgE, or Amb-APE-specific IgE in supernatants from anti-CD40/IL-4/ Amb-APE-stimulated B cells (data not shown). We then investigated whether factors of Amb-APE other than allergens (or proteins in general) might cause the IgE enhancement. Therefore, murine C57BL/6 B cells were incubated under Th2 conditions with Amb-APE total, Amb a 1, with an allergen-free fraction of Amb-APE ( $<3 \mathrm{kDa})$, or with the pollen-associated compounds E1 phytoprostane (PPE1) and prostaglandin E2 (PGE2). To exclude an effect of LPS contamination of pollen on IgE induction, we also incubated with LPS. The major ragweed allergen Amb a 1 (Fig. 3A), LPS (Fig. S1), and PGE2 (data not shown) did not affect IgE levels, but the low-molecular-weight fraction enhanced IgE production to a similar extent as total Amb-APE (Fig. 3A). Also, the pollen-associated mediator PPE1 (Fig. 3B) increased IgE in a dose-dependent manner. Thus, IgE enhancement by Amb-APE does not require the major ragweed allergen or other proteins, but is elicited by lowmolecular-weight substances such as PPE1.

\section{APEs from various plant species enhance IgE production}

Because PPE1 had also been detected in birch pollen (3), we investigated whether the in vitro $\operatorname{IgE}$ enhancement is a common effect of pollen extracts independent from plant species. Therefore, we compared the effects of pollen extracts from a variety of plant species. Besides Amb-APE, we tested pollen extracts from birch, timothy grass, and pine.

Extracts of ragweed (Amb-APE), birch (Bet-APE), timothy grass (Phl-APE), and pine pollen (Pin-APE) were used as

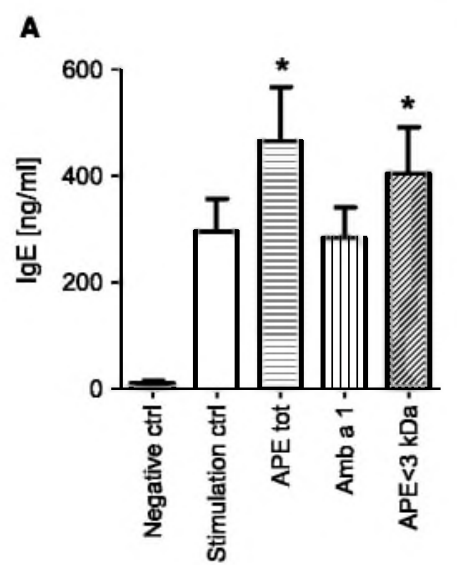

B

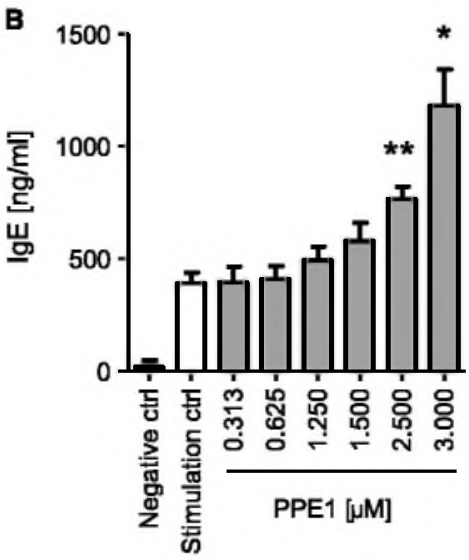

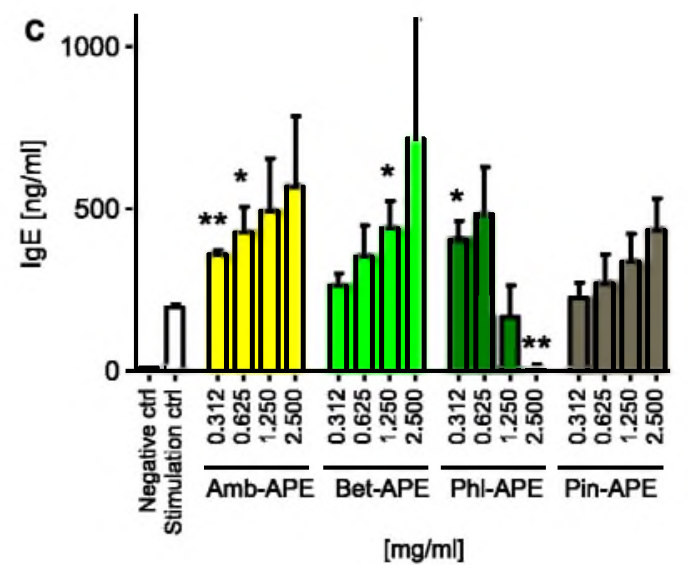

Figure $3 \lg E$ enhancement caused by allergen-free pollen fraction, allergen-free compound PPE1, and other pollen species. Naîve B cells from C57BL/6 mice were stimulated with anti-CD40 and IL-4. (A) IgE enhancement by $1.25 \mathrm{mg} / \mathrm{ml}$ of Amb-APE, allergen-free APE fraction (Amb-APE $<3 \mathrm{kDa}$ ), but not by Amb a 1 alone (same concentration as in $1.25 \mathrm{mg} / \mathrm{ml}$ Amb-APE). (B) IgE enhancement by PPE1. (C) IgE enhancement by pollen extracts from ragweed (AmbAPE), birch (Bet-APE), grass (Phl-APE), and pine (Pin-APE). Data are displayed as mean $+\mathrm{SD}$. $n=3-4$ independent experiments. ${ }^{*} P \leq 0.05,{ }^{*} P \leq 0.01$ compared to stimulation control. 
adjuvant in the anti-CD40/IL-4 B-cell stimulation assay (C57BL/6). Besides Amb-APE, also Bet-APE, Phl-APE, and Pin-APE dose-dependently increased IgE production (Fig. 3C). Low-dose Phl-APE augmented IgE production, but led to lower IgE secretion at concentrations of 1.25 $2.5 \mathrm{mg} / \mathrm{ml}$. A highly statistically significant $(P<0.01) \operatorname{IgE}$ increase was detected for anti-CD40/IL-4/Amb-APE costimulation, while this effect was significant but less pronounced $(P<0.05)$ also with Bet-APE and Phl-APE and was reversed at higher concentrations of Phl-APE. Pin-APE showed a similar tendency to enhance IgE from Th2-primed murine B cell. Therefore, the IgE-enhancing effect of pollen extracts is not specific to ragweed pollen, but is also caused by pollen from various plant species, including trees and grasses.
IgE enhancement by pollen extracts depends on B-cell proliferation

To investigate the mechanism of APE-induced augmentation of $\operatorname{IgE}$ production, we first evaluated immunoglobulin isotype switching in B cells, as it is a prerequisite for IgE production. We measured transcripts of IgE CSR by quantitative realtime PCR following aCD40/IL-4 \pm Amb-APE stimulation of C57BL/6 naïve B cells. The IgE CSR-facilitating transcripts AID and $\varepsilon$-GLT were not induced upon Amb-APE exposure compared to stimulation control, but the $\varepsilon$-postswitch transcript (IgE precursor) was up-regulated by Amb-APE (Fig. 4A). Thus, Amb-APE did not enhance IgE CSR, but increased the expression of $\operatorname{IgE}$ transcript. We further investi-

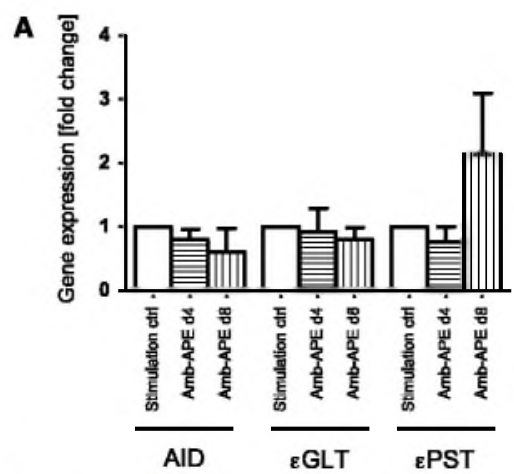

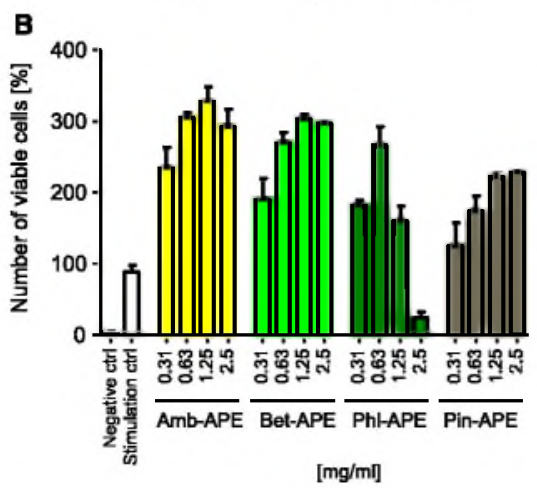
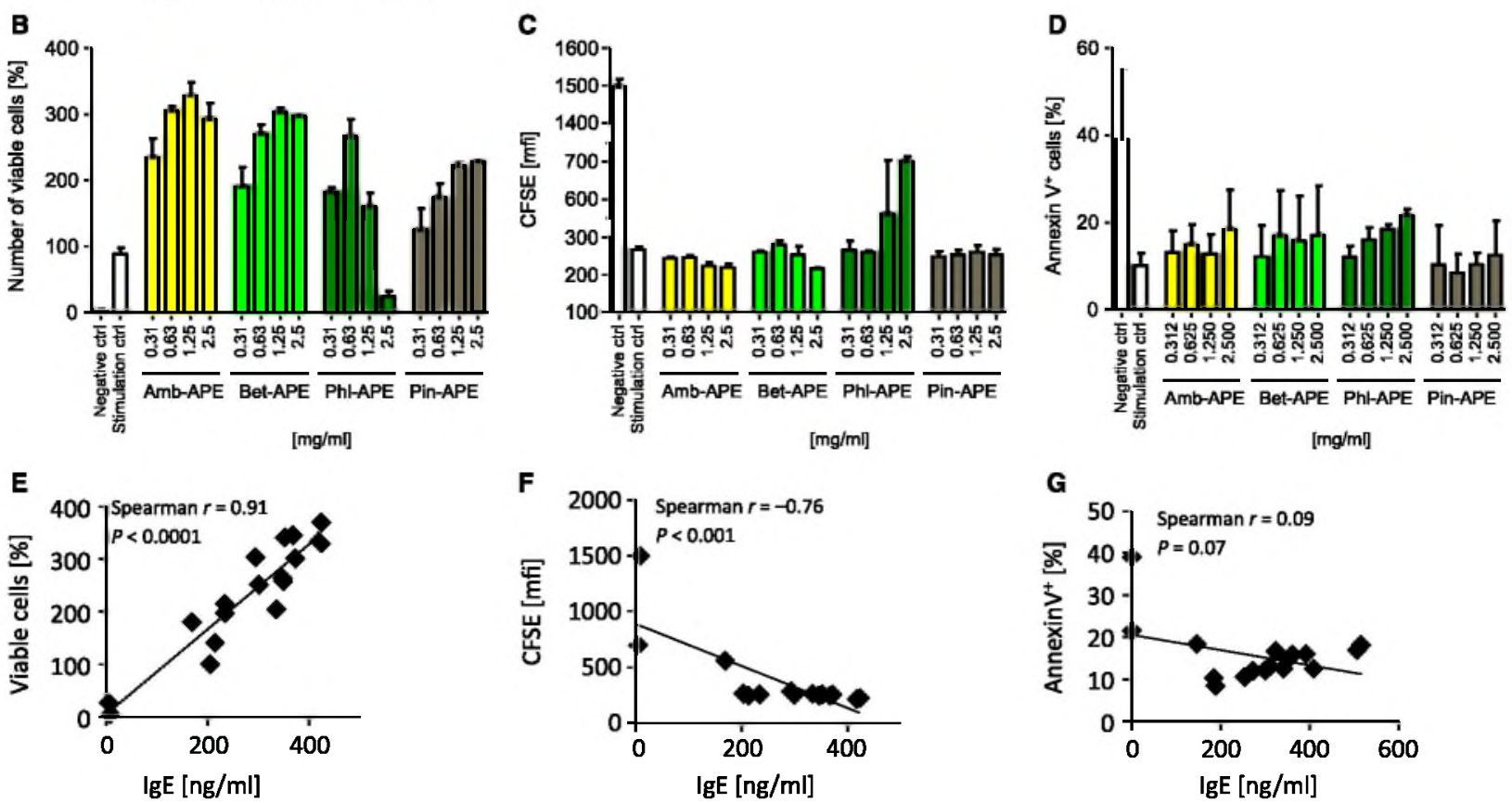

G

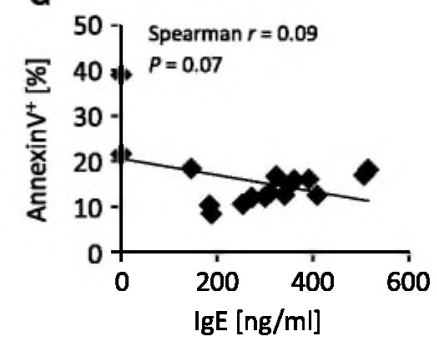

Figure 4 Mechanism of pollen-induced lgE augmentation. Naïve B cells from C57BL/6 mice were stimulated with anti-CD40 and IL-4 together with or without pollen extracts. (A) Expression of IgE class switch-associated transcripts AID lactivation-induced cytidine deaminase), $\varepsilon-G L T$ ( $\varepsilon$ germline transcript), and the precursor of $\lg E$ transcript E-PST (e-postswitch transcript) in B cells 0,4 , and 8 days after stimulation. Relative mRNA expression levels were calculated using the $2^{-\Delta \Delta C t}$ method. HPRT: housekeeping gene. (B) Number of viable $B$ cells on day 8 measured by intracellular ATP content. (C) Proliferation of $B$ cells measured as mean fluorescence intensity (mfi) of CFSE. (D) Proportion of apoptotic cells (7-AAD /annexin $\left.\mathrm{V}^{+}\right)$. $(E, F, G)$ correlation with $\lg E$ level in supernatant for $B$, $C$, and D, respectively. Data are expressed as mean $+\mathrm{SD} . n=3$ (A) and 1 representative experiment (B-G). 
gated whether the pollen extract-induced increase in IgE production was correlated with the number of $\mathbf{B}$ cells at the end of the experiment. Count of viable B cells was assessed by measuring intracellular ATP on day 8 of culture following increasing concentrations of APEs. The number of viable cells increased dose dependently with APE exposure (Fig. 4B) and followed the same pattern as the $\operatorname{IgE}$ production (Fig. 3C). Correlation of cell count and IgE levels was highly significant ( $P<0.0001$; correlation coefficient: 0.91 [Fig. 4E]). Because higher number of viable cells can be achieved by either increased proliferation or decreased apoptosis, both mechanisms were analyzed. Proliferation of CFSE-stained B cells showed the same coherency with IgE levels (Fig. 4C/F). In contrast, apoptosis (annexin V staining) did not correlate with IgE production (Fig. 4D/G).

\section{Human memory B cells increase IgE production after PPE1} exposure

To translate our findings into the human system, we stimulated human naïve and memory B cells from healthy

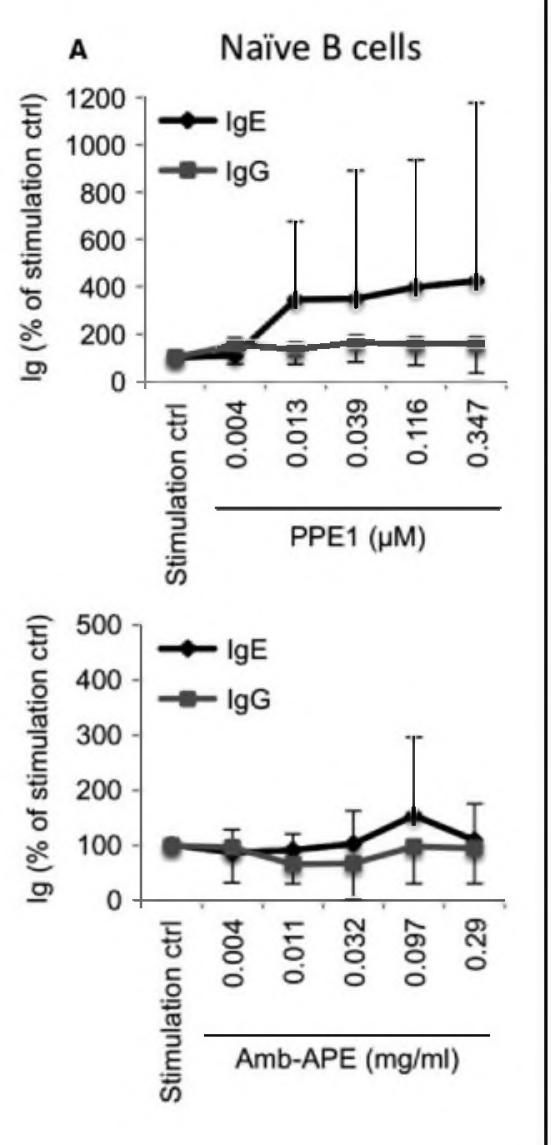

Figure 5 Modulation of immunoglobulin secretion in human naive and memory B cells by PPE1 and Amb-APE. Naïve (A) and memory (B) B cells were stimulated with IL-4 and IL-21 and incubated with increasing concentrations of PPE1 and Amb-APE. Displayed are IgE and IgG concentrations in B-cell supernatants as percentage of stim- donors $(n=6)$ with increasing concentrations of PPE1 and Amb-APE together with IL-4, IL-21, and SCD40L (Fig. 5). In naive B cells, the response to PPE1 demonstrated high donor variability, and there was no significant effect of Amb-APE (Fig. 5A). A significant increase in IgE secretion was observed in memory B cells after incubation with PPE1, while IgG secretion remained unchanged with increasing concentrations of PPE1 (Fig. 5B). Incubation of memory B cells with Amb-APE induced a slight increase in IgE secretion, which did not reach statistical significance (Fig. 5B).

\section{Discussion}

Pollen extracts showed the ability to enhance $\operatorname{IgE}$ production in both the in vitro Th2-like stimulated B cells and an in vivo OVA-sensitization model. This IgE enhancement depended on an increase in B-cell proliferation and number, but not of IgE class switch recombination. Causing factors, such as PPE1, were present in the protein-free fraction of ragweed pollen extracts.

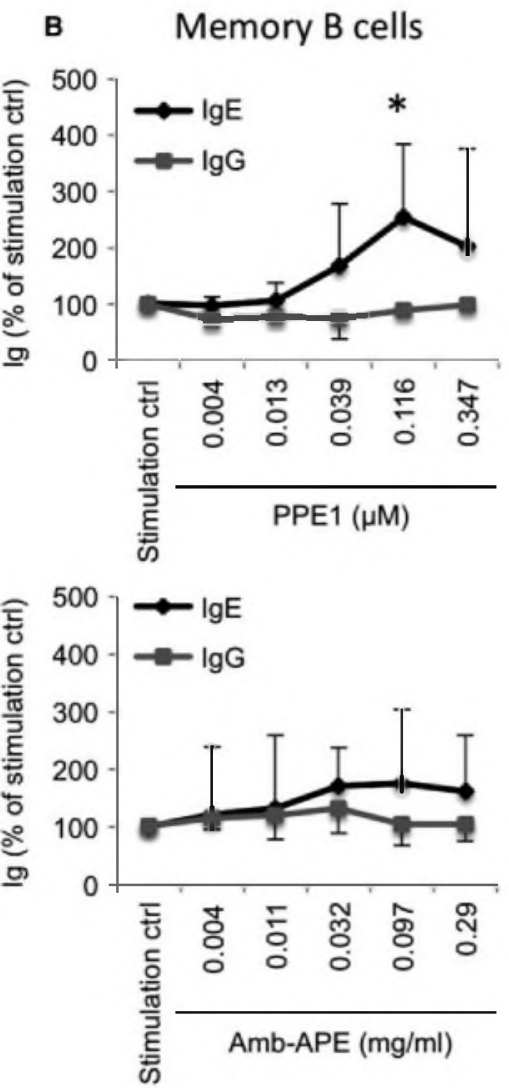

ulation control (mean $+\mathrm{SD}$ ). $n=5-6$ donors. Absolute values of stimulation control: naive B cells: $44.9 \mathrm{ng} / \mathrm{ml}$ lgE, $27699170 \mathrm{ng} / \mathrm{ml}$ lgG; memory B cells $27.1 \mathrm{ng} / \mathrm{ml} \mathrm{lgE，87616} 650 \mathrm{ng} / \mathrm{ml} \mathrm{lgG}$. ${ }^{*} P<0.05$ compared to stimulation control. 
Pollen extracts alone or under Th1-like conditions did not affect immunoglobulin production, while the polyclonal Ig secretion under Th2-like conditions was enhanced. The increase was specific to the allergy-associated antibody isotype IgE. Using an OVA mouse model, we demonstrated an Amb-APE-induced IgE increase with an adjuvant effect of ragweed pollen, which was characterized by an early recruitment of BAL macrophages, lymphocytes, and eosinophils, that increased further in the later time-point. The adjuvant effect of ragweed was also confirmed in the histological analysis by an increased inflammatory infiltrate and mucus hypersecretion.

The pollen extract-enhanced IgE production is mediated by a variety of plant pollen including those of weeds, grass, and trees and even pine, which are very low in protein but high in lipid content (29). Together with the observation that only nonspecific IgE production was measurable upon AmbAPE exposure, this lead to the hypothesis that substances of the pollen extracts other than allergens or proteins are sufficient to enhance IgE production. In fact, incubation with a protein-/allergen-free fraction containing low-molecularweight substances caused the same enhancing effects as observed with total pollen extract. In contrast, the major allergen of ragweed Amb a 1 applied in the same concentration as present in Amb-APE did not influence IgE production. Low-molecular-weight substances [PALMs, (2)] were suitable candidates with adjuvant activity. PALM $\mathrm{E}_{1}$-phytoprostanes signal via PPAR- $\gamma(8)$, which enhances B-cell antibody production and differentiation (30). Our results show for the first time that PPE1 has a direct influence on B cells by enhancing ongoing IgE production. A further candidate substance might be LPS, which is described to enhance Th2 responses in low concentrations (31) and in combination with IL-4 to induce CSR to IgE (32). In our pollen extracts, LPS was measurable in low concentrations (data not shown), but LPS without pollen extract did not increase IgE production when applied together with anti-CD40 and IL-4 (Fig. S1). Thus, LPS is not related to pollen-enhanced IgE production. The allergen alone is not only unnecessary for $\operatorname{IgE}$ enhancement, but can even have the opposite effect. In human memory B cells, a grass pollen allergen-mediated inhibition of IgE production has been reported via increased IL-10 production (33). This observation supports our finding that other substances of the pollen extracts than the allergen itself, such as nonallergenic proteins or small molecules like PPE1, can exert the IgE-enhancing effect.

The mechanism of APE-mediated enhancement of IgE production seems to be an increased proliferation rate in B cells that switch their immunoglobulin isotype toward IgE. This effect was detected with pollen extracts from four different plant species. However, there are also plant species, like Typha angustata (cattail), that produce pollen compounds with antiproliferative properties (34). In contrast, rapeseed pollen-derived polysaccharides were found to increase the proliferation of lymphocytes (35). While proliferation of IgEgenerating B cells was increased in vitro, IgE class switch recombination itself was not. Takhar et al. (14) found that allergen exposure directly induces local IgE class switch recombination outside of lymphoid tissue. Local IgE CSR was found in noses of allergic rhinitis patients and in bronchial mucosa of asthmatics, but not in atopic patients without asthma (13). Thus, local IgE CSR seems to be dependent on a specific local micromilieu. In certain patients, the IgEenhancing effects of pollen that we found in our experiments might then be potentiated by an additional increase in IgE CSR.

Pollen-derived phytoprostanes have structural similarity to endogenous prostaglandins that were previously found to increase IgE in B cells, such as PGE2 (36). PGE2-induced $\mathrm{IgE}$ enhancement was caused by an increase in the proportion of IgE-secreting B cells (37) caused by enhanced IgE CSR that depends on increased levels of $\operatorname{AID}(37,38)$. In our system, CSR was not affected and PGE2 did not influence IgE synthesis.

In our human B-cell experiments, we evaluated the effect of Amb-APE and PPE1 on both naïve and memory B cells. We observed high donor variability, especially in naïve $B$ cells. This might be due to the small number of cells per well used in the experiments. Therefore, small differences in the cell number might have a massive effect on the IgE production.

Because the effect of the pollen extracts is higher in memory B cells compared to nonswitched naïve B cells, we hypothesize that these pollen-derived substances have a stronger effect on proliferation of preexisting IgE-switched memory B cells than they have on class switch recombination toward IgE in naïve B cells, similarly to what was seen in mouse. It is plausible that the higher concentration of $\operatorname{IgE}$ in memory B cells might also simply arise from preexisting IgEswitched B cells in this fraction.

This study provides for the first time data on the direct influence of pollen extracts on B cells that result specifically in increased $\operatorname{IgE}$ secretion. This finding further emphasizes the impact of pollen-derived nonallergenic low-molecularweight substances in allergic sensitization and inflammation and might be of special importance for patients suffering from local allergic rhinitis.

\section{Acknowledgments}

We thank Alexandra Seisenberger, Johanna Grosch, Benjamin Schnautz, and Katja Haslauer for their excellent technical assistance. We thank Dr. C.H. Heusser for human IL-4.

\section{Funding}

Christine Kühne-Center for Allergy Research and Education (CK Care), Davos (S.O., F.A., M.W., U.F., S.G., C.T.H., H.B.). KKF C grant 8761150 , Faculty of Medicine, Technische Universität München (J.G.). Swiss National Science Foundation 320030_140772 (M.A.)

\section{Conflicts of interest}

The authors have declared no conflict of interest. 


\section{Supporting Information}

Additional Supporting Information may be found in the online version of this article:

Figure S1. IgE-enhancing effect of Amb-APE is not LPSdependent. Naive B cells were stimulated with anti-CD40/IL$4 \pm$ LPS. On day 8 total IgE (A), IgG (B), and IgM (C) were determined. Data are shown as medians \pm quartiles and maximum/minimum. $n=5-7$ independent experiments.
Figure S2. Titration of B cells for optimal IgE-secretion. Titration of naïve and memory B cells was done starting with 25000 cells down to 195 cells per well in a 96 -well plate. IgE secretion was induced by stimulation with human CD40-ligand-presenting mouse fibroblasts, $25 \mathrm{ng} / \mathrm{ml}$ human IL-21 and human IL-4 and measured by ELISA.

Table S1. Primers used for real-time PCR.

Data S1. Materials and Methods.

\section{References}

1. Wierenga EA, Snoek M, de Groot $\mathrm{C}$, Chretien I, Bos JD, Jansen HM et al. Evidence for compartmentalization of functional subsets of CD2 + T lymphocytes in atopic patients. J Immunol 1990;144:4651-4656.

2. Behrendt H, Kasche A, Ebner von Eschenbach C, Risse U, Huss-Marp J, Ring J. Secretion of proinflammatory eicosanoid-like substances precedes allergen release from pollen grains in the initiation of allergic sensitization. Int Arch Allergy Immunol 2001;3:121-125.

3. Traidl-Hoffmann C, Mariani V, Hochrein H, Karg K, Wagner H, Ring J et al. Pollenassociated phytoprostanes inhibit dendritic cell interleukin- 12 production and augment T helper type 2 cell polarization. $J$ Exp Med 2005;201:627-636.

4. Traidl-Hoffmann $\mathbf{C}$, Jakob T, Behrendt $\mathbf{H}$. Determinants of allergenicity. $J$ Allergy Clin Immunol 2009;123:558-566.

5. Miyagawa F, Gutermuth J, Zhang H, Katz SI. The use of mouse models to better understand mechanisms of autoimmunity and tolerance. $J$ Autoimmun 2010;35:192198.

6. Traidl-Hoffmann C, Kasche A, Jakob T, Huger M, Plotz S, Feussner I et al. Lipid mediators from pollen act as chemoattractants and activators of polymorphonuclear granulocytes. $J$ Allergy Clin Immunol 2002; 109:831-838.

7. Plotz SG, Traidl-Hoffmann C, Feussner I, Kasche A, Feser A, Ring J et al. Chemotaxis and activation of human peripheral blood eosinophils induced by pollen-associated lipid mediators. $J$ Allergy Clin Immunol 2004;113:1152-1160

8. Gilles S, Mariani V, Bryce M, Mueller MJ, Ring J, Jakob T et al. Pollen-derived E1phytoprostanes signal via PPAR-gamma and NF-kappaB-dependent mechanisms. $J$ Immu nol 2009;182:6653-6658.

9. Gutermuth J, Bewersdorff M, Traidl-Hoffmann C, Ring J, Mueller MJ, Behrendt $\mathrm{H}$ et al. Immunomodulatory effects of aqueous birch pollen extracts and phytoprostanes on primary immune responses in vivo. $J$ Allergy Clin Immunol 2007;120:293-299.
10. Mariani V, Gilles S, Jakob T, Thiel M, Mueller MJ, Ring J et al. Immunomodulatory mediators from pollen enhance the migratory capacity of dendritic cells and license them for Th2 attraction. $J$ Immunol 2007;178:7623-7631

11. Boldogh I, Bacsi A, Choudhury BK, Dharajiya N, Alam R, Hazra TK et al. ROS generated by pollen NADPH oxidase provide a signal that augments antigen-induced allergic airway inflammation. $I$ Clin Invest 2005;115:2169-2179.

12. Vinhas R, Cortes L, Cardoso I, Mendes VM, Manadas B, Todo-Bom A et al. Pollen proteases compromise the airway epithelial barrier through degradation of transmembrane adhesion proteins and lung bioactive peptides. Allergy 2011;66:1088-1098.

13. Takhar $P$, Corrigan CJ, Smurthwaite L, O'Connor BJ, Durham SR, Lee TH et al Class switch recombination to IgE in the bronchial mucosa of atopic and nonatopic patients with asthma. $J$ Allergy Clin Immunol 2007;119:213-218.

14. Takhar P, Smurthwaite L, Coker HA, Fear DJ, Banfield GK, Carr VA et al. Allergen drives class switching to IgE in the nasal mucosa in allergic rhinitis. $J$ Immunol 2005; 174:5024-5032.

15. Rondon C, Campo $P$, Galindo L, BlancaLopez N, Cassinello MS, Rodriguez-Bada $\mathrm{JL}$ et al. Prevalence and clinical relevance of local allergic rhinitis. Allergy 2012;67:12821288.

16. Rondon C, Campo P, Togias A, Fokkens WJ, Durham SR, Powe DG et al. Local allergic rhinitis: concept, pathophysiology, and management. $J$ Allergy Clin Immunol 2012;129:1460-1467.

17. Okazaki IM, Kinoshita K, Muramatsu $\mathbf{M}$, Yoshikawa $\mathrm{K}$, Honjo T. The AID enzyme induces class switch recombination in fibrob lasts. Nature 2002;416:340-345.

18. Muramatsu M, Kinoshita $K$, Fagarasan $S$, Yamada S, Shinkai Y, Honjo T. Class switch recombination and hypermutation require activation-induced cytidine deaminase (AID), a potential RNA editing enzyme. Cell 2000;102:553-563.
19. Geha RS, Jabara HH, Brodeur SR. The regulation of immunoglobulin E class-switch recombination. Nat Rev Immunol 2003;3:721-732

20. Iciek LA, Delphin SA, Stavnezer J. CD40 cross-linking induces Ig epsilon germline transcripts in $\mathrm{B}$ cells via activation of NF-kappaB: synergy with IL-4 induction J Immunol 1997;158:4769-4779.

21. Warren WD, Berton MT. Induction of germ-line gamma 1 and epsilon Ig gene expression in murine $B$ cells. IL- 4 and the CD40 ligand-CD40 interaction provide distinct but synergistic signals. $J$ Immunol 1995;155:5637-5646.

22. Poulsen LK, Hummelshoj L. Triggers of IgE class switching and allergy development. $A n n$ Med 2007;39:440-456.

23. Gilles S, Fekete A, Zhang X, Beck I, Blume $\mathrm{C}$, Ring J et al. Pollen metabolome analysis reveals adenosine as a major regulator of dendritic cell-primed $\mathrm{T}(\mathrm{H})$ cell responses. $J$ Allergy Clin Immunol 2011;127:454-461.

24. Leonard R, Wopfner N, Pabst M, Stad1mann J, Petersen BO, Duus JO et al. A new allergen from ragweed (Ambrosia artemisiifolia) with homology to art $\mathrm{v} 1$ from mugwort J Biol Chem 2010;285:27192-27200.

25. Wopfner N, Jahn-Schmid B, Schmidt G, Christ T, Hubinger G, Briza $P$ et al. The alpha and beta subchain of Amb a 1, the major ragweed-pollen allergen show divergent reactivity at the $\mathrm{IgE}$ and T-cell level. Mol Immunol 2009;46:2090-2097.

26. Alessandrini F, Beck-Speier I, Krappmann D, Weichenmeier I, Takenaka S, Karg E et al. Role of oxidative stress in ultrafine particle-induced exacerbation of allergic lung inflammation. Am J Respir Crit Care Med 2009;179:984-991

27. Braun A, Engel T, Aguilar-Pimentel JA, Zimmer A, Jakob $T$, Behrendt $\mathbf{H}$ et al. Beneficial effects of cannahinoids (CB) in a murine model of allergen-induced airway inflammation: role of $\mathrm{CB} 1 / \mathrm{CB} 2$ receptors Immunobiology 2011;216:466-476.

28. Avery DT, Ma CS, Bryant VL, SantnerNanan B, Nanan R, Wong $M$ et al. STAT3 is required for IL-21-induced secretion of 
IgE from human naive B cells. Blood 2008;112: 1784-1793.

29. Jato MV, Rodriguez FJ, Seijo MC. Pinus pollen in the atmosphere of Vigo and its relationship to meteorological factors. Int $J$ Biometeorol 2000;43:147-153.

30. Garcia-Bates TM, Baglole CJ, Bernard MP, Murant TI, Simpson-Haidaris PJ, Phipps RP. Peroxisome proliferator-activated receptor gamma ligands enhance human $\mathbf{B}$ cell antibody production and differentiation. $J$ Immunol 2009;183:6903-6912.

31. Eisenbarth SC, Piggott DA, Huleatt JW, Visintin I, Herrick CA, Bottomly $\mathrm{K}$.

Lipopolysaccharide-enhanced, toll-like receptor 4-dependent T helper cell type 2 responses to inhaled antigen. $J$ Exp Med 2002;196: 1645-1651
32. Kashiwada M, Levy DM, McKeag L, Murray $\mathrm{K}$, Schroder $\mathrm{AJ}$, Canfield SM et al. IL4-induced transcription factor NFIL3/E4BP4 controls IgE class switching. Proc Natl Acad Sci USA 2010;107:821-826.

33. Milovanovic $M$, Heine $G$, Zuberbier $T$, Worm M. Allergen extract-induced interleukin-10 in human memory $B$ cells inhibits immunoglobulin E production. Clin Exp Allergy 2009;39:671-678.

34. Nhiem NX, Kiem PV, Minh CV, Lee JJ, Ku $\mathrm{JH}$, Myung CS et al. A potential inhibitor of rat aortic vascular smooth muscle cell proliferation from the pollen of Typha angustata. Arch Pharm Res 2010;33:19371942

35. Yang X, Guo D, Zhang I, Wu M. Characterization and antitumor activity of pollen polysaccharide. Int Immunopharmacol 2007;7:427-434.

36. Roper RL, Conrad DH, Brown DM, Warner GL, Phipps RP. Prostaglandin E2 promotes IL-4-induced IgE and IgG1 synthesis. $J$ Immunol 1990;145 2644-2651

37. Roper RL, Brown DM, Phipps RP. Prostaglandin E2 promotes B lymphocyte Ig isotype switching to IgE. $J$ Immunol 1995;154:162-170.

38. Lee H, Trott JS, Haque S, McCormick S, Chiorazzi N, Mongini PK. A cyclooxygenase-2/prostaglandin E2 pathway augments activation-induced cytosine deaminase expression within replicating human B cells. J Immunol 2010;185: $5300-5314$ 